\title{
LA CONCILIACIÓN EN MATERIA DE FAMILIA COMO FACTOR DE ACCESO A LA ADMINISTRACIÓN DE JUSTICIA EN EL CENTRO DE CONCILIACIÓN DE CECAR, SINCELEJO, SUCRE, AÑOS 2016 A 2018
}

\author{
Gina Paola Arroyo Jaramillo ${ }^{1}$ \\ Daniela Montes Arrieta ${ }^{2}$ \\ Luisa Fernanda Vásquez Cotera ${ }^{3}$
}

\section{Resumen}

La familia como base de la sociedad muchas veces enfrenta ciertos conflictos que pueden atentar contra la unidad, el respeto y la tolerancia que antes la caracterizaba, entonces se hace necesario lograr la solución de esas controversias sin obstáculos que compliquen los casos y es allí donde la conciliación como mecanismo alternativo de solución de conflictos les permite a los miembros de la familia la construcción de esa solución. Por otro lado, en el ejercicio conciliatorio es la conciliación en materia de familia la que con mayor frecuencia se presenta y es en las familias de escasos recursos donde los centros de conciliación gratuitos recobran gran importancia, pues les permiten acceder a la administración de justicia mediante un mecanismo caracterizado por la celeridad, la eficiencia, y la gratuidad. Se planteó en la presente investigación realizar un análisis sobre la conciliación en materia de familia como factor de acceso a la administración de justicia en el Centro de Conciliación de la Corporación Universitaria del caribe CECAR, Sincelejo en los años 2016 a 2018, cuyas estadísticas demuestran que el Centro de Conciliación recibió un importante número de solicitudes, de las cuales la mayoría concluyeron en un acuerdo total sobre las

$1 \quad$ Especialista en Derecho Procesal Civil de la Corporación Universitaria del Caribe CECAR. Email: ginna.arroyoj@cecar.edu.co

2 Especialista en Derecho Procesal Civil de la Corporación Universitaria del Caribe CECAR. Email: daniela daniela.montes@cecar.edu.co

3 Especialista en Derecho Procesal Civil de la Corporación Universitaria del Caribe CECAR. Email: luisa.vasquezc@cecar.edu.co 
pretensiones, que este servicio se prestó en un mínimo de tiempo y garantizando de manera oportuna los derechos de los solicitantes, especialmente los constitucionales de administración de justicia y debido proceso.

Palabras clave: Conciliación, Derecho de Familia, Administración de Justicia, Centros de Conciliación, Eficacia de la Conciliación, Mecanismos Alternativos de Solución de Conflictos.

\section{Abstract}

The family as the basis of society often faces certain conflicts that can undermine the unity, respect and tolerance that once characterized it, so it is necessary to achieve the resolution of such disputes without obstacles that complicate the cases and it is there where conciliation as an alternative dispute resolution mechanism allows family members to build such a solution. On the other hand, in the conciliation exercise it is the family conciliation that most frequently occurs and it is in low-income families that free conciliation centres regain great importance, since they allow them access to the administration of justice through a mechanism characterized by celerity, efficiency and gratuity. It was proposed in the present research to conduct an analysis of family conciliation as a factor in access to the administration of justice at the Conciliation Center of the Caribbean University Corporation CECAR, Sincelejo in the years 2016 to 2018, whose statistics show that the Conciliation Center received a significant number of requests, most of which concluded in a total agreement on the claims, that this service was provided in a minimum of time and timely guaranteeing the rights of applicants, especially the constitutional rights of administration of justice and due process.

Keywords: Conciliation, Family law, administration of justice, conciliation centres, conciliation effectiveness, alternative dispute resolution mechanisms.

\section{Introducción}

Colombia fue el primer país en Latinoamérica en otorgar un rango constitucional a la conciliación y en legislar en dicha área, lo que fue un avance significativo, ya que hasta antes de la Constitución de 1991 sólo el Estado estaba facultado para administrar justicia, pero, con la promulgación 
de la nueva Carta su artículo 116 estipuló que "los particulares pueden ser investidos transitoriamente de la función de administrar justicia en la condición de jurados en las causas criminales, conciliadores o en la de árbitros habilitados por las partes para proferir fallos en derecho o en equidad. Según lo establecido en la Constitución Política de, lo que desmonopolizó la administración de justicia. En 1998 se definió por conducto de la ley la conciliación, como "un mecanismo de resolución de conflictos a través del cual, dos o más personas gestionan por sí mismas la solución de sus diferencias, con la ayuda de un tercero neutral y calificado, denominado conciliador", de acuerdo a la Ley, definición que posteriormente fue incorporada por el Decreto 1818 de 1998, el cual regula los mecanismos alternativos de solución de conflictos. Cabe recordar que la conciliación puede ser judicial o extrajudicial, de acuerdo a si se realiza o no dentro de un proceso judicial, en este trabajo se tratará la conciliación extrajudicial y específicamente en materia del derecho de familia, como una forma de disminuir la violencia, ya que los problemas familiares muchas veces son muy complicados y requieren una orientación más pacífica para mitigar la desunión familiar.

Partiendo de este contexto se estudiará la conciliación como figura jurídica y su incidencia en el acceso a la administración de justicia, dado que es imprescindible cultivar en la sociedad colombiana una cultura de diálogo para la resolución de sus conflictos y mitigar de esta forma el contexto violento donde la sociedad se ha visto inmerso durante siglos. Reiteradamente se ha mencionado por muchos doctrinantes nacionales que el sistema de administración de justicia está colapsado y que hay muchos casos que no ameritan activar todo el aparato judicial cuando pueden ser resueltos por otras figuras propias del derecho, una de dichas figuras es la conciliación, al contar nuestra facultad con un centro de conciliación se nos hizo relevante dar a conocer el trabajo de éste en la sociedad sincelejana, así como hacer un análisis de la conciliación como mecanismo alternativo de solución de conflicto. Por otro lado, partiendo del hecho de que la sociedad colombiana y en particular la sucreña ha sido duramente golpeada por el conflicto armado interno, la cual ha dejado como consecuencias que el tejido social se haya fragmentado y marcado por la violencia, se hace ineludible el fomentar la aplicación de figuras que no coloquen a las partes en condición de contrincantes, donde resulta un ganador y un vencedor, sino en un plano de igualdad donde ambos a través del diálogo puedan resolver sus controversias sin la existencia de roles de ganadores y perdedores. Finalmente, resulta enriquecedor 
para los estudiantes de derecho y abogados el apropiarse de esta figura jurídica para así lograr que no sea necesario acudir a instancias judiciales para llegar a la solución de los asuntos de familia, o lograr que una vez activado el aparato jurisdiccional se puedan para evacuar en la mayor brevedad posible los casos. Se ha planteado como objetivo general el Determinar la eficacia conciliación en materia de familia como factor de acceso a la administración de justicia en el centro de conciliación de CECAR, Sincelejo - Sucre durante los años 2016 a 2018, lo cual se desarrollara al conceptualizar y analizar los mecanismos alternativos de solución de conflictos como factor de acceso a la administración de justicia desde una perspectiva doctrinal, jurisprudencial y normativa, identificar a través del análisis estadístico la eficacia de la conciliación en materia de familia del Centro de Conciliación de la Facultad de Derecho de la Corporación Universitaria del Caribe CECAR en Sincelejo - Sucre y establecer el aporte de los centros de conciliación como factor de acceso a la administración de justicia.

La presente investigación busca dar a conocer la conciliación como un mecanismo alternativo de solución de conflicto y como una figura judicial creada por el legislador en el artículo 116 constitucional referente a los particulares investidos transitoriamente para administrar justicia ya que partiendo de que la justicia en el país es la sexta más lenta del mundo por lo cual el mismo legislador reconoció que los particulares pueden entrar a colaborar en la administración de justicia para así cumplir con el principio de eficiencia y celeridad que la misma Carta pregona, además, tiene como propósito analizar los índices de utilización y eficacia de la conciliación y siendo la conciliación gratuita en materia de familia en los consultorios jurídicos, le permite a personas de escasos recursos una verdadera entrada al acceso a la administración de justicia, con el fin de darle respuesta a la pregunta problema planteada ¿Cuál es la eficacia de la conciliación en materia de familia como factor de acceso a la administración de justicia en el centro de conciliación de CECAR, Sincelejo - Sucre durante los años 2016 a 2018 ?

\section{Mecanismos Alternativos de Solución de Conflictos MASC}

Los mecanismos alternativos de solución de conflictos son una apuesta diferente a la justicia tradicional, es decir, una justicia alternativa, y que se adaptan a las necesidades de la sociedad, permitiendo una administración de justicia pronta, que no requiere la representación judicial de un abogado y que en el ámbito del posconflicto colombiano contiene figuras como la 
conciliación que son mecanismos que optan por la resolución pacífica de las controversias.

Ahora, el tratamiento que se le da a nivel doctrinal, jurisprudencial y legal es realmente enriquecedor para estos. Muchos han sido los doctrinantes que se han expresado sobre los mecanismos alternativos de solución de conflictos, como lo es Becerra (2009), quien menciona que:

Los mecanismos alternativos de solución de conflictos son una vía alterna a la justicia formal o procesal, eminentemente voluntaria, de impartir justicia de corte restaurativo, lo que genera un cambio en la concepción del derecho de acceso a la justicia, y con una finalidad más encaminada a beneficiar a las víctimas, aunado a los esfuerzos por parte de los entes estatales de administrar justicia de forma efectiva. (p.271)" (Cabana, G, 2017, p.8)

Entonces, partimos de comprender los MASC como una forma concomitante de administrar justicia, la forma tradicional en la que interviene el Estado o un tercero que era quien tomaba la decisión, representa precisamente una forma anticuada y violenta de solucionar los conflictos, de una perspectiva más autónoma, voluntaria y pacífica surgen estos mecanismos que tienen como finalidad permitir que los implicados en un conflicto conversen y converjan una solución, a la vez que van disminuyendo la carga del Estado en la administración de justicia.

Por su parte, el Centro de Conciliación y Consultorio Jurídico de la Universidad Cooperativa de Colombia pregona que los MASC son posibilidades que tienen las personas en conflicto para solucionar las controversias sin intermediación de un juez o un proceso judicial, por lo cual, consagran una manera más ágil, amistosa, sencilla, eficaz y eficiente de resolver los conflictos con plenos efectos legales. Además, resalta el papel del conciliador como un tercero capacitado para llevar el diálogo, para crear la atmósfera donde las partes puedan discutir sus puntos de vista y proponer soluciones.

Centrándose en la conciliación, expresa que es "mecanismo de solución de conflictos a través del cual, dos o más personas gestionan por sí mismas la solución de sus diferencias, con la ayuda de un tercero neutral y calificado, denominado conciliador." Podemos observar que las diferencias conceptuales no son muchas, pues es la práctica y la regulación legal de cada mecanismo la que los diferencian 
sustancialmente. Doctrinariamente, se puede concluir que los MASC son una alternativa a la justicia formal y tradicional, a los cuales pueden acceder todas las personas para buscar una solución amistosa donde no existan ganadores o vencedores, sino un desenlace dado en el punto medio de dicha controversia. Las partes que hacen uso de estos mecanismos poseen una amplia gama de posibilidades para resolver los putos en enfrentamiento de forma expedita y eficaz.

La Corte Constitucional en su basta jurisprudencia ha plasmado la importancia que tienen los MASC en la solución de los conflictos que se presentan entre particulares, resumiéndolos así: "i) buscan hacer efectivo uno de los fines constitucionales como el de la convivencia pacífica; (ii) permiten la participación directa de los interesados en la resolución de sus conflictos; (iii) son otra forma de hacer efectivo el derecho de acceso a la administración de justicia, y (iv) son un buen mecanismo para lograr la descongestión judicial, conforme lo establecido en la Sentencia Constitucional de 2013. Partiendo de lo anterior, la Corte resalta que la Conciliación en particular es un mecanismo que permite a las personas acceder a la administración de justicia, pero, no solo como parte en la cual busca dirimir sus conflictos jurídicos, sino también cuando funge como conciliador.

Por otro lado, se ha reconocido que los MASC, especialmente la conciliación, plantean un mecanismo de autocomposición que permite resolver las controversias con agilidad y con costos inferiores a la justicia tradicional y formal, por lo anterior, promueve la convivencia pacífica, respetando el debido proceso, reduciendo las dilaciones injustificadas en el proceso de solución y hace más efectiva la administración de justicia como servicio público.

Hemos hablado mucho de administración de justicia, pero alcanzamos a entender iqué es? La misma Corte Constitucional ha reiterado que es un derecho fundamental que se relaciona directamente con el derecho al debido proceso, y que comprende los medios idóneos y efectivos para la protección de los derechos y obligaciones. Igualmente, plantea que el tiempo de resolución de los conflictos debe ser prudente para evitar la violación de otros derechos de las personas, $y$, principalmente, debe garantizar que existan mecanismos que proporcionen una real administración de justicia especialmente a las partes pobres que no pueden costear el acceso a la justicia formal, es aquí cuando los MASC se consolidan como una verdadera oferta de justicia, una respuesta operativa y efectiva para asegurar ese derecho. 
Se ha avalado que la constitucionalidad de los MASC recae sobre el deber del Estado y los particulares de contribuir al mantenimiento de la paz social, así como también busca hacer realidad los principios y valores que el ordenamiento jurídico y el Estado Social de Derecho pregonan, entre esos, la paz, la tranquilidad, el orden justo y la armonía de las relaciones sociales. En este sentido se ha reiterado que <para la Corte es claro que esas metas se hacen realidad no sólo mediante el pronunciamiento formal y definitivo de un juez de la República, sino que asimismo es posible lograrlo acudiendo a la amigable composición o a la intervención de un tercero que no hace parte de la rama judicial. Se trata, pues, de la implementación de las denominadas "alternativas para la resolución de los conflictos", conforme lo establecido en la Sentencia Constitucional de 2016, pues la finalidad de la justicia formal y de estos mecanismos es llegar a una solución a las diferencias que se suscitan entre los particulares, siendo los mecanismos una respuesta más pacífica y menos compleja.

Finalmente, se reconoce que los MASC constituyen un instrumento importante para la descongestión de los despachos judiciales y la efectiva administración de justicia.

No existe una norma que regule todos los mecanismos alternativos de solución de conflictos, sino que en distintas leyes se han estipulado las disposiciones referentes a cada mecanismo. Es así como la amigable composición y el arbitraje son regulados en la ley 1563 de 2012, definiendo:

Arbitraje: artículo 1.- es el mecanismo alternativo de solución de conflictos mediante el cual las partes trasladan a árbitros la solución de una controversia relativa a asuntos de libre disposición o aquellos que la ley autorice. Mientras la amigable composición: artículo 59.- es el mecanismo por medio del cual, dos o más particulares, éstos y una o más entidades públicas, o varias entidades públicas, o quien desempeñe funciones administrativas, delegan en un tercero, denominado amigable componedor, la facultad de definir, con fuerza vinculante para las partes, una controversia contractual de libre disposición.

La mediación por otra parte, se encuentra regulada en el Código de Procedimiento Civil y en las sentencias C-381 de 1996 y C1195 de 2001 de la Corte Constitucional, y se define como el mecanismo donde un tercero neutral, particular o servidor público designado ya 
sea por el Fiscal General de la Nación o su delegado busca permitir un intercambio de opiniones entre la víctima y el imputado o acusado para que confronten sus puntos de vista y, con su intervención, logren solucionar el conflicto que les enfrenta. La conciliación se encuentra regulada por la Ley 640 de 2001, sin embargo, esta ley no la define como MASC, pero si regula la aplicación de dicha figura a la vez que la clasifica en judicial o extrajudicial, dependiendo si se realiza dentro del marco de un proceso judicial o no. Es esta misma ley la que en su artículo 11 plasma la existencia de los centros de conciliación en consultorios jurídicos de facultades de derecho, donde se admiten a los estudiantes de derecho como conciliadores cuando los asuntos sean de cuantías de competencia de los consultorios jurídicos o como auxiliares de los abogados que funjan como conciliadores cuando la cuantía sea superior a la estipulada para consultorios jurídicos, es así como se observa que la conciliación, y los MASC tienen su asidero en la ley, que permite una mejor aplicación de las figuras.

\section{La eficacia de la conciliación en materia de familia del Centro de Conciliación de la Facultad de Derecho de la Corporación Universitaria del Caribe CECAR en Sincelejo}

Inicialmente, cabe rescatar que la conciliación ha sido reconocida por su importancia en la construcción de soluciones pacíficas, así como ha permitido a los particulares encontrar una respuesta rápida, económica, justa, eficaz y sin dejar perdedores entre las partes cuando se presentan conflictos, y es que la familia como institución base de la sociedad se encuentra con controversias propias que sólo se presentan al interior de ésta, como lo son los alimentos, las sociedades conyugales y patrimoniales, la custodia de los hijos y su respectivo régimen de visitas, entre otras, donde la conciliación puede ofrecer a las partes la construcción de una solución.

La conciliación fue definida por la Ley 446 de 1998 en su artículo 64, clasificándola como un "mecanismo de resolución de conflictos donde dos o más personas gestionan por sí mismas la solución de sus diferencias, con la ayuda de un tercero neutral y calificado llamado conciliador." Además de los interesados en el enfrentamiento, citante y citado, interviene un tercero capacitado que es el conciliador, éste solo es un guía de las partes, una persona que puede proponer fórmulas de arreglo para que los interesados avancen en la construcción de su solución, también, es vigía para que el 
diálogo sea constructivo y el encargado de plasmar los acuerdos a los que se haya llegado en el acta de conciliación.

Ahora bien, ese tercero imparcial, por disposición de la Ley 640 de 2001, puede ser un estudiante de derecho adscrito al centro de conciliación de una facultad de derecho. Estos centros de conciliación tienen la finalidad de promover el acceso a la administración de justicia y son un servicio que las universidades ofrecen a la población de menos recursos. La Corporación Universitaria del caribe CECAR en su Consultorio Jurídico de la Facultad de Derecho cuenta con una dependencia que es el Centro de Conciliación que tiene como misión crear y fortalecer la consciencia democrática y tolerante para el desarrollo de una comunidad justa y equitativa. Desde 2016 el Centro de Conciliación ha realizado 926 procesos conciliatorios, de los cuales 606 han sido en materia de familia.

\section{Centro de Conciliación \\ CECAR}

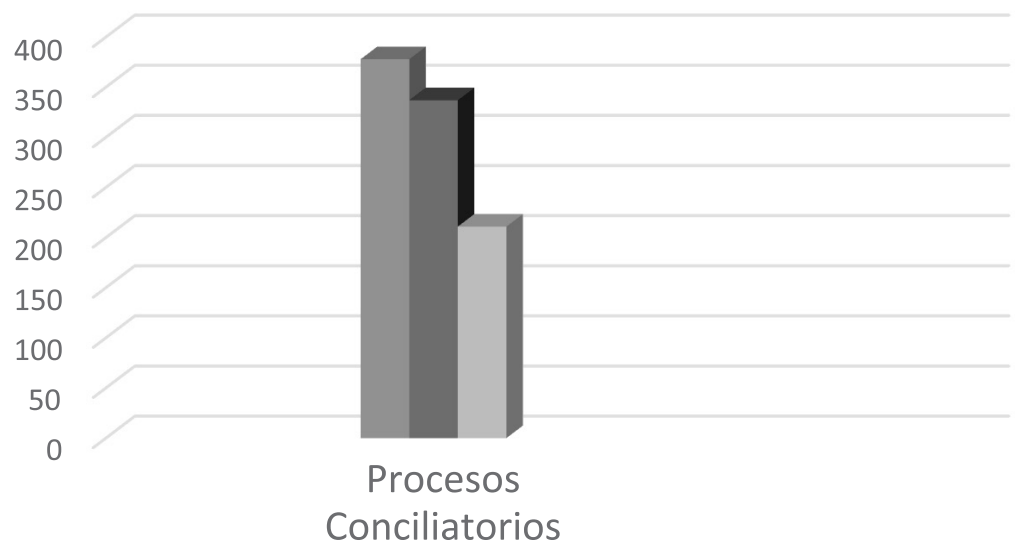

\section{6 - 2017 2018-I}

Gráfica 1. Procesos Conciliatorios del Centro de Conciliación CECAR 20162018.

Creación propia. Fuente Centro de Conciliación - CECAR (Año 2018)

De los 926 procesos que se presentaron, 428 lograron llegar a un acuerdo total, mientras que sólo 110 no llegaron a ningún acuerdo y 1 realizó acuerdo parcial. Igualmente, se expidieron 298 constancias de no asistencia de alguna de las partes y 47 con un resultado distinto; 42 de las solicitudes fueron retiradas. 
Observándose una mayor proporción en los casos donde las partes llegan a un acuerdo respecto a los casos donde no. Además, se observa que la inasistencia a la audiencia de conciliación es frecuente, lo que no representa un porcentaje significativo frente al número de casos donde las personas sí se someten al proceso conciliatorio, lleguen o no a una solución, ya que son 298 casos de inasistencia, es decir, un 33.71\%, respecto a los 586 casos representativos del 66.28\%.

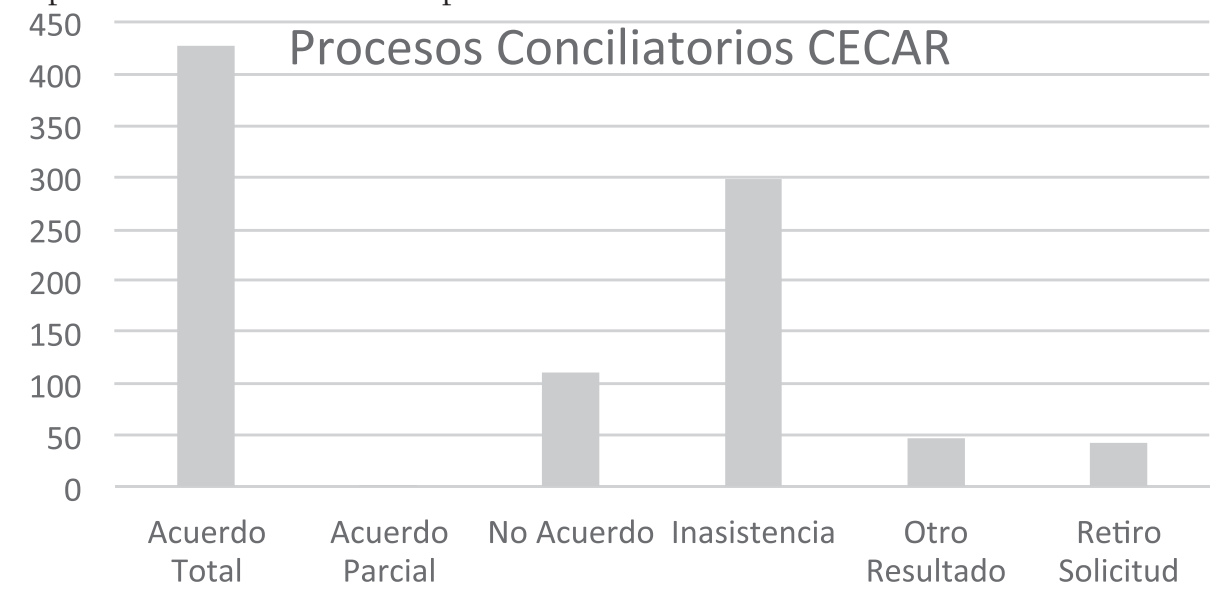

\section{Procesos Conciliatorios}

Gráfica 2. Resultado Procesos Conciliatorios del Centro de Conciliación - CECAR

Creación propia. Fuente Centro de Conciliación - CECAR (Año 2018)

De los 926 procesos conciliatorios se refleja que 609 son en materia de familia, es decir, que las conciliaciones en esta área representan el $65.9 \%$ del total. Esto se debe en medida a que son personas de escasos recursos quienes acceden a los servicios del centro, solicitando muchas veces la protección de los derechos de sus hijos, como los alimentos y las visitas de los padres, igualmente, porque la justicia formal requiere una inversión monetaria importante para que se de la administración de justicia, los costos del abogado, los trámites y demás son elevados frente a la condición socioeconómica de estas familias, además, se requiere que sea expedita, pronta, y la congestión judicial en el país no garantiza que sea resuelto un asunto en un mínimo de tiempo, como sí lo permite la conciliación, pues son las mismas partes las que deciden.

En los 609 procesos conciliatorios en materia de familia, 334 lograron un acuerdo total, mientras que 63 no conciliaron y 1 realizó un acuerdo parcial. Así mismo, fueron 168 los casos de inasistencia, 24 los 
resultados diferentes y 19 los que retiraron la solicitud. Es decir, que un $56.61 \%$ de los casos expuestos en el Centro de Conciliación llegaron a un acuerdo sobre todas las pretensiones; un $10.67 \%$ no lograron construir una solución; el 4.06\% llegó a un resultado distinto y el 0.16\% realizó una conciliación parcial respecto a las pretensiones.

En cuanto a la inasistencia de alguna de las partes, representa un $28.47 \%$ de los procesos, mientras que el retiro de la solicitud de conciliación es del 3.22\%, siendo mínimos los casos donde se desiste de iniciar el proceso de conciliación.

\section{Procesos Conciliatorios en Familia}

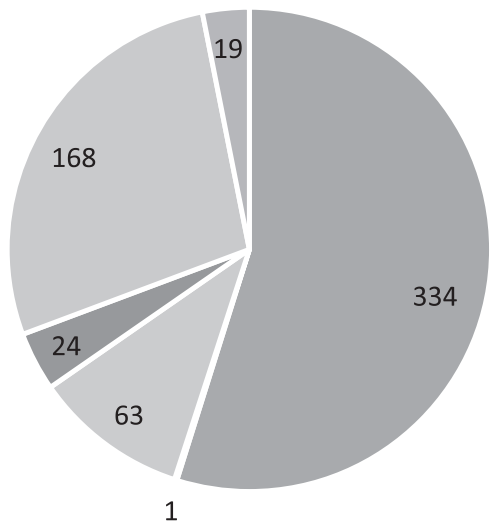

Acuerdo Total Acuerdo Parcial No Acuerdo Otro Resultado Inasistencia Retiro

\section{Gráfica 3. Procesos Conciliatorios en Familia.}

Creación propia. Fuente Centro de Conciliación - CECAR (Año 2018).

Se puede observar que el Centro de Conciliación ha llevado en su mayoría procesos conciliatorios satisfactorios, donde las partes han encontrado soluciones en derecho de forma gratuita, ágil y eficaz. De esta manera, la corporación ha promovido el uso de los mecanismos alternativos de solución de conflictos, ha administrado justicia de forma transitoria teniendo como base la comunicación, influyó en la descongestión de los despachos judiciales del distrito judicial, fomenta la resolución pacífica de las controversias, ejerce de forma transitoria una función jurisdiccional y ha contribuido a la convivencia pacífica entre los ciudadanos de la región. 


\section{Aporte de los centros de conciliación como factor de acceso a la administración de justicia}

El acceso administración de justicia se ha reconocido por la jurisprudencia colombiana como la posibilidad que tienen las personas de solicitar que un juez competente proteja sus derechos, además, este derecho también contempla la oportunidad de acudir a los mecanismos alternativos de solución de conflicto para dirimir las diferencias que se les presenten a los particulares. Esta doble concepción del derecho a la administración de justicia parte del supuesto de que tanto el Estado a través de sus jueces, como los funcionarios de los MASC, son muestras de "procedimientos idóneos y efectivos para la determinación legal de los derechos y las obligaciones", y al estar el Estado obligado a promover este tipo de espacios que permitan la protección de dicho derecho constitucional, se facultó a las facultades de derecho para contar con un centro de conciliación adscrito a su consultorio jurídico, con el fin de lograr una garantía efectiva de acceso a la administración de justicia y en la búsqueda la realización máxima de este derecho.

La obligación legal de las universidades de establecer centros de conciliación tiene un doble matiz, por un lado, permite a los estudiantes de las facultades de derecho capacitarse en MASC y de practicar el rol de conciliador; así como también tiene la finalidad de que las universidades presten el servicio de forma gratuita, lo que incluye un enfoque diferencial donde a las personas de escasos recursos se les está constituyendo espacios idóneos para la protección de su derecho a la administración de justicia, es por eso que la población a la cual se dirigen estos centros son las de escasos recursos.

Partiendo del trabajo de campo y de la revisión teórica del derecho al acceso a la administración de justicia por parte de los centros de conciliación de las universidades, se observó que:

1. El componente de gratuidad de estos centros de conciliación ha permitido que un mayor número de personas puedan acceder a la administración de justicia, especialmente la población de menores recursos, permitiéndoles el goce efectivo de sus derechos. Esta es una de las políticas públicas que ha tenido en cuenta el estado de vulnerabilidad de esta población y se ha enfocado en suplir en cierta medida el déficit de acceso a la justicia formal a través de unos mecanismos sencillos y eficaces.

2. Permite la participación de los afectados en la construcción de la solución, pues son los mismos interesados los que llegan a 
un acuerdo o deciden si acogen las propuestas del conciliador o no, es decir, siempre es la voluntad de las partes la que determinará el resultado de la conciliación.

3. Fomenta la convivencia pacífica al promover un mecanismo amistoso donde las partes a través del diálogo dirimen sus diferencias, lo cual en el contexto violento en el que ha estado sumido Colombia es un gran avance hacia la reconstrucción del tejido social y la propagación de una sociedad en paz.

4. Ha permitido a las personas acceder de forma ágil y eficaz a un medio de resolución de sus conflictos, ya que en comparación con la justicia formal, los MASC son un apuesta a la rapidez, ya que mientras medie la voluntad de las partes, la solución es pronta y oportuna.

5. Tiene consecuencias judiciales en caso de incumplimiento, pues si alguna de las partes incumple con los acuerdos pactados, la parte afectada puede dirigirse a la justicia formal para hacer valer el contenido del acta de conciliación.

6. Por último, ha permitido descongestionar el aparato de justicia, ya sea por su uso de forma independiente o como un requisito de procedibilidad para ingresar a un proceso judicial, el cual ha sostenido la Corte es un fin legítimo ya que el "sistema judicial está al borde del colapso por el gran número de procesos que impide su pronta resolución a pesar de la actividad de los jueces." Lo que incide de manera directa en la efectividad del servicio público de administración de justicia.

\section{Conclusión}

Finalmente, a través del estudios de los mecanismos alternativos de solución de conflictos, el trabajo del centro de conciliación de la Corporación Universitaria del Caribe - CECAR, y los aportes que la conciliación ha realizado, se puede determinar que el trabajo de conciliación en derecho de familia como medio alterno de solución de controversias de este centro ha contribuido a que las personas del círculo de Sincelejo accedan a la administración de justicia contribuyendo a la descongestión de esta sede judicial.

Ultimando, las estadísticas demuestran que durante los años 2016 a 2018 el centro de conciliación recibió importante número de solicitudes, de las cuales la mayoría concluyeron en un acuerdo 
total sobre las pretensiones, que este servicio se prestó en un mínimo de tiempo y garantizando de manera oportuna los derechos de los solicitantes, especialmente los constitucionales de administración de justicia y debido proceso. Por otra parte, es el derecho de familia el que más presenta solicitudes para procesos conciliatorios, lo cual radica en parte en las condiciones socioeconómicas de la población a la que se dirige este servicio.

Es de resaltar la labor realizada por el centro de conciliación de la Corporación, que demuestra el compromiso social que tiene con la comunidad sincelejana y sucreña, así como con el cumplimiento de los deberes legales que se le han asignado, siendo la promoción y fomento de la conciliación una herramienta para la construcción de paz social, para la protección de los derechos de las personas y para la capacitación idónea de sus estudiantes de derecho.

\section{Referencias}

Cabana, M. A. (20 de SEPTIEMBRE de 2017). DE LOS MECANISMOS ALTERNATIVOS DE SOLUCION DE CONFLICTOS EN COLOMBIA: ACERCA DE SU ALCANCE Y DESARROLLO PARA SU IMPLEMENTACION EN LOS MUNICIPIOS DE POST-CONFLICTO. Obtenido de UNIVERSIDAD CATÓLICA: https://repository.ucatolica.edu.co/bitstream/10983/14606/1/DE\%20LOS\%20MECANISMOS\%20ALTERNATIVOS\%20DE\%20SOLUCION\%20DE\%20 CONFLICTOS\%20EN\%20COLOMBIA\%20\%281\%29.pdf

CÓDIGOGENERALDELPROCESO,LEY1564(CONGRESODELAREPÚBLICA 12 de JULIO de 2012).

COlOMBIA, U. C. (2 de OCTUBRE de 2018). Centro de Conciliación y Consultorio Jurídico. Obtenido de Centro de Conciliación y Consultorio Jurídico: https://www.ucc.edu.co/arauca/Paginas/centro-de-conciliacion-y-consultorio-juridico.aspx

CONSTITUCIÓNPOLÍTICA, 1991(ASAMBLEACONSTITUYENTE20deJULIO de 1991). 
Estatuto de Arbitraje Nacional e Internacional y se dictan otras disposiciones, Ley 1563 de 2012 (CONGRESO DE LA REPÚBLICA 12 de JULIO de 2012).

ESTATUTODELOSMECANISMOSALTERNATIVOSDESOLUCIÓNDECONFLICTOS,DECRETO 1818(MINISTERIODEJUSTICIAYDELDERECHO 7 de SEPTIEMBRE de 1998).

LEY, 446 (CONGRESO DE LA REPÚBLICA 7 de JULIO de 1998).

LEYSOBRELACONCILIACIÓN, LEY640(CONGRESODELAREPÚBLICA5 de ENERO de 2001).

Sentencia Constitucional, Sentencia C-222 (CORTE CONSTITUCIONAL 2013).

SENTENCIADECONSTITUCIONALIDAD, SentenciaC-404de2016(CORTE CONSTITUCIONAL DE COLOMBIA 2016).

SENTENCIADETUTELA, T-1044(CORTECONSTITUCIONAL22 deOCTUBRE de 2004). 